\title{
Comparison of two strategies for handgrip force prediction based on SEMG
}

\author{
Hongxin Cao \\ College of Computer Science and Technology \\ Zhejiang University \\ Hangzhou, China \\ chxin@zju.edu.cn \\ Shouqian Sun \\ College of Computer Science and Technology \\ Zhejiang University \\ Hangzhou, China \\ ssq@zju.edu.cn \\ Zenggui Gao \\ College of Computer Science and Technology \\ Zhejiang University \\ Hangzhou, China \\ gzg@zju.edu.cn
}

\author{
Chao Li \\ College of Computer Science and Technology \\ Zhejiang University \\ Hangzhou, China \\ superli@zju.edu.cn \\ Weixin Wang \\ College of Computer Science and Technology \\ Zhejiang University \\ Hangzhou, China \\ wwx515@sina.com \\ Xiaogang Zhang \\ College of Computer Science and Technology \\ Zhejiang University \\ Hangzhou, China \\ zxgs11@126.com
}

\begin{abstract}
The control system of myoelectric prostheses requires high precision and rapid response. Many algorithms have been applied in prosthesis control. In this paper, BackPropagation Neural Network (BPNN) and Multiple Nonlinear Regression (MNLR) are applied to predict handgrip force through surface electromyography (sEMG) signals of forearm muscles. In the following experiments, the root mean square (RMS) data extracted from sEMG signals are randomly separated into training dataset $(\mathbf{7 5 \%})$ and testing dataset $(25 \%)$. When the dataset is trained, the Root Mean Square Error can reach about $1.145 \mathrm{kfg}$ (BPNN) and $3.452 \mathrm{kfg}$ (MNLR), respectively. BPNN consumes about 21.435s and MNLR spends about 0.013s. During testing the dataset, BPNN and MNLR obtain the Root Mean Square Error about $1.207 \mathrm{kfg}$ and $3.620 \mathrm{kfg}$, respectively. BPNN consumes nearly the same time with MNLR. Based on the comparison of BPNN and MNLR, BPNN outperforms MNLR at accuracy, and MNLR is better than BPNN at response time. This study results will provide an important basis for the reasonable selection of prosthetic wrist system.
\end{abstract}

Keywords-handgrip force; sEMG; RMS; BPNN; MNLR

\section{INTRODUCTION}

Nowadays, more and more individuals are disabled by industrial accidents, diseases and armed conflicts. Many myoelectric prostheses are used to assist the paralyzed individuals and compensate for the lost capabilities of the upper extremities [1]-[3]. Handgrip force prediction is important for controlling prostheses. In previous studies, surface EMG signal is often selected for handgrip force prediction because it can reflect muscle activity directly. Back-propagation artificial neural networks and statistical regression analysis are applied to estimate handgrip force
[4][8]. The good relationships among sEMG signals, handgrip force and joint parameters were obtained using artificial neural networks [4]. Grip force was successfully estimated from integrated sEMG by two layered artificial neural network [5]. Some linear regression models indicated the valid estimation of handgrip force from sEMG signals [6][7]. J.Duque et al. applied multiple nonlinear regression to analyze the relationship between handgrip force and sEMG signals. The excellent relativity between measured force and estimated force was obtained [8].

In this paper, BPNN and MNLR are applied to map handgrip force signals onto sEMG signals off-line. RMS values extracted from sEMG signals are used as input data for BPNN and MNLR. BPNN is evaluated by comparing its performances (accuracy, correlation and response time) with MNLR. Then it can be decided that which strategy is suitable for the control system of myoelectric prostheses at different occasions.

The remainder of this paper is organized as follows: two strategies used in this study are introduced briefly in Section II. After the experiments are described in Section III, Signal processing and evaluation indexes are presented in Section IV. The results are discussed in Section V. Finally, the conclusion and future work will be mentioned in Section VI.

\section{METHODS}

\section{A. BPNN}

BPNN is very effective for learning and calculating from mixtures of signals without too much detailed information [9]. BPNN has been applied in biological 
fields, acting as a black box model to approximate complex nonlinear mappings directly from input values [10]. In this study, BPNN contains three layers (input layer, hidden layer and output layer). According to the Kolmogorov theorem, the number of hidden layer's nodes is set to thirteen. BPNN works in following sections according to the flowchart (Fig .1).

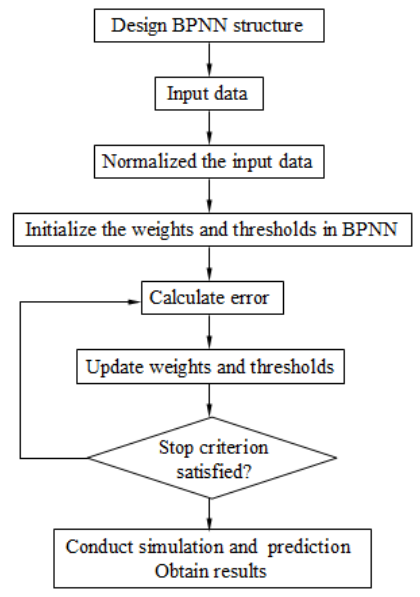

Figure 1. The flowchart of BPNN.

\section{B. $M N L R$}

Regression analysis is a method of making an interpretation when the value of one variable is defined as a function of the observed values of other variables. It can be defined as the estimated or predicted value of one variable from the values of other given variables [11][12]. Principal component analysis (PCA) is a technique that is useful for reducing the number of predictive variables and solving the multi-colinearity problems [13][14]. PCA looks for a few linear combinations of the variables that can be used to summarize the original data without losing too much information, that is, preserve as much information as possible. Details about PCA can be seen in [15][16]. In this study, the dimensionality of the input data is reduced through the PCA method. The MNLR works in the following sections according to the flowchart (Fig .2).

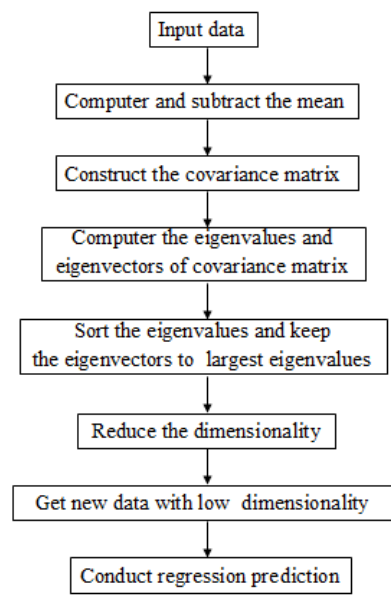

Figure 2. The flowchart of PCA.

\section{EXPERIMENTS}

In this study, ten participants are recruited (age $=23 \pm 3$ years, weight $=61.6 \pm 8.0 \mathrm{~kg}$ ). They all apply the dominant right hand to handgrip force. All the participants had an examination to eliminate upper extremity complaints and give informed consent prior to the experiments.

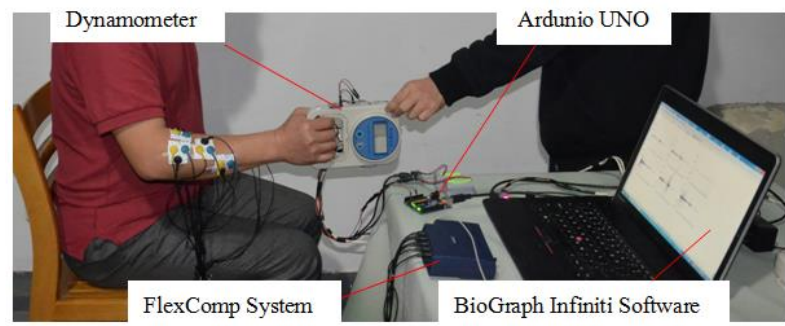

Figure 3. The arm and hand posture in this study.

According to the American Society of Hand Therapists (ASHT) [17][18], every participant put his wrist at the neutral position and hold a modified dynamometer (WCS1000, Shanghai Wanqing Electron Co.,Ltd) (Fig .3). The data of handgrip force is transmitted to the computer from the dynamometer by a microcomputer (Ardunio UNO). The sEMG signals are obtained by FlexComp System with EMG MyoScan modules and EMG electrodes (Thought Technology Ltd. in Canada), then sEMG signals are recorded and analyzed by BioNeuro Infiniti software during experiments [19].

Six forearm muscles are selected for study. These muscles are Brachioradialis (BR), Flexor Carpi Radialis (FCR), Flexor Carpi Ulnaris (FCU), Extensor Carpi Radiali (ECR), Extensor Carpi Ulnaris (ECU), Extensor Digitorum (ED). All these muscles, locating in the outermost layer, are available for sEMG recording. Every participant is required to conduct grip tasks following the curve profiles. As shown in Fig .4, the curve profiles from zero to MVC force with a duration time (about 8s) are performed.

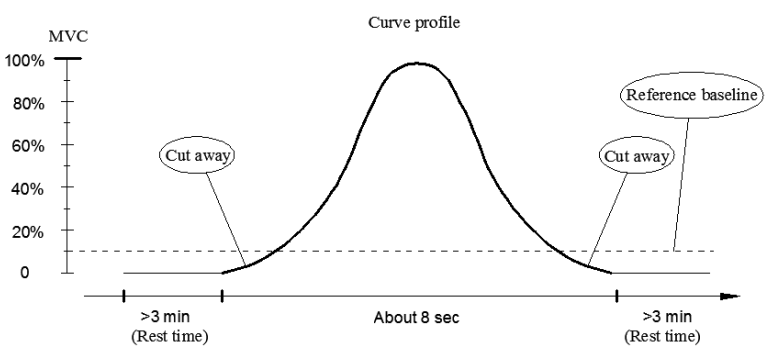

Figure 4 . The reference baseline and the curve profile.

\section{SIGNALS PROCESSING AND EVALUATION INDEXES}

\section{A. Signals processing}

For the curve profile of handgrip force, ten percent of MVC forces are extracted as its reference baseline. Then the curve profile is cut by its reference baseline. All the input and output data of two methods are normalized to [-1 1] in this paper [19][20]. The forces from the processed curve profiles are normalized by its MVC force using Equation (1).

$$
F^{\prime}=2 \times\left(F-{ }_{\text {min }} F\right) /\left({ }_{\max } F-{ }_{\text {min }} F\right)-1
$$

Where $F^{\prime}$ is the normalized data, $F$ is the measured and processed data, $\min F$ is the minimum value in measured dataset, $\max F$ is the maximum value in measured dataset, the minimum value in normalized range is set to negative 1 , the maximum value in normalized range is set to positive 1 . 

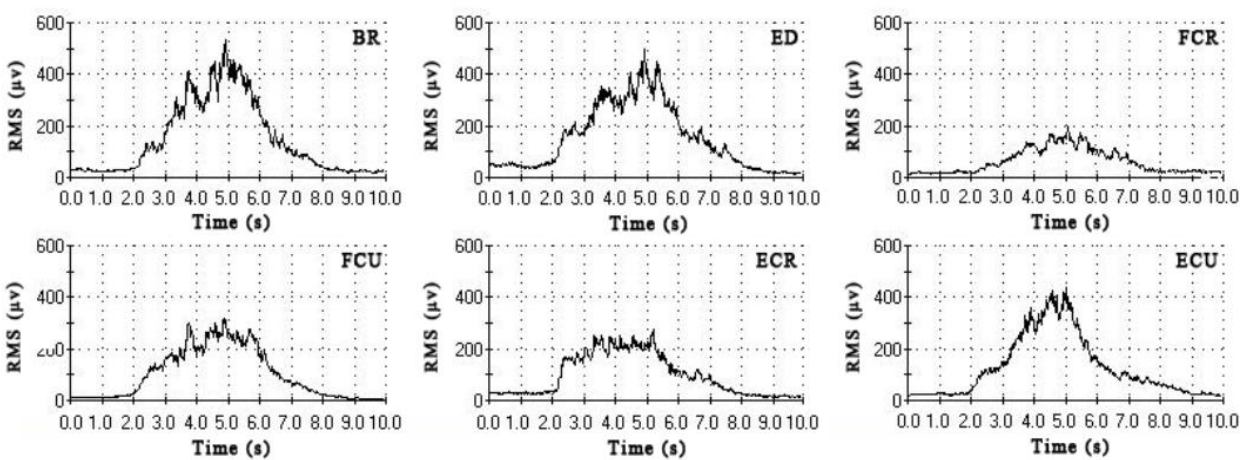

Figure 5. RMS signals from one participant

The sEMG signals are sampled at $1024 \mathrm{~Hz}$. A band pass filter $(20 \mathrm{~Hz} \sim 500 \mathrm{~Hz})$ [6] and a notch filter $(50 \mathrm{~Hz})$ [21] are applied to remove unnecessary contaminated noises in the recorded sEMG signals. RMS features are extracted from sEMG signals. Raw RMS signals from one participant are shown in Fig .5. Corresponding to handgrip force, RMS curves are cut by itself reference baselines (twenty percent of max RMS value). Then the remaining RMS signals are sub-sampled to the sampling frequency of handgrip force. The processed RMS signals are normalized using Equation (2)

$$
r^{\prime}=2 \times\left(r-{ }_{\text {min }} R\right) /\left({ }_{\max } R-{ }_{\text {min }} R\right)-1
$$

Where $r$ ' is the normalized RMS data, $r$ is the measured and processed RMS data, $\min R$ is the minimum RMS value in measured dataset, $\max R$ is the maximum RMS value in measured dataset, the minimum RMS value in normalized range is set to negative 1 , the maximum RMS value in normalized range is set to positive 1 .

\section{B. Evaluation indexes}

After the processing of RMS signals and handgrip force signals, the algorithm methods are selected for training and testing the data. During the experiments, the data from the cut curve profile are randomly separated into training dataset $(75 \%)$ and testing dataset $(25 \%)$. The performances of two strategies are evaluated by the Root Mean Square Error (RMSE), Correlation Coefficient $(C C)$ and Consuming Time/1 run $(C T)$.

The RMSE is achieved using Equation (3).

$$
R M S E=\sqrt{\left[\sum_{\mathrm{i}=1}^{\mathrm{n}}\left(f_{\mathrm{i}}-F_{\mathrm{i}}\right)^{2}\right] / N}
$$

Where $f_{\mathrm{i}}$ is the predicted value, $F_{\mathrm{i}}$ is the measured value, $N$ is the number of handgrip force in the dataset.

The $C C$ is achieved using Equation (4).

$$
C C=\operatorname{Cov}(f, F) / \sqrt{\operatorname{Var}(f) \cdot \operatorname{Var}(F)}
$$

Where $\operatorname{Cov}(f, F)$ is the covariance of predicted value and measured value, $\operatorname{Var}(f)$ is the variance of predicted value, $\operatorname{Var}(F)$ is the variance of measured value.

In order to obtain the comparison results, firstly, BPNN and MNLR are trained for many times (100 times) separately based on training dataset. All the RMSE values (100 times) are averaged for every participant in training dataset and testing data. Then all the averaged $R M S E$ from the participants are taken to Mean \pm Standard Deviation $\left(\operatorname{Mean}_{R M S E} \pm\right.$ S.D.RMSE $)$. The Mean $\operatorname{MMSE}_{\text {S S.D. }}$ RMSE acts as one of evaluation indexes for BPNN and MNLR. In the same way, the processed $C C\left(\operatorname{Mean}_{C C} \pm\right.$ S.D. $\left.{ }_{C C}\right)$ and the consuming time/1 run $\left(\operatorname{Mean}_{C T} \pm\right.$ S.D. $\left.{ }_{C T}\right)$ are taken as evaluation indexes of BPNN and MNLR.

\section{RESULTS AND DISCUSSIONS}

After the experiments are conducted and the signals are processed, the results are shown in Table 1 and Table 2, respectively.

\begin{tabular}{|c|c|c|c|}
\hline Method & $\operatorname{Mean}_{R M S} \pm$ S.D.RMSE $(k f g)$ & Mean $_{C C} \pm$ S.D. $C C$ & $\operatorname{Mean}_{C T} \pm$ S.D..$_{C T}(s)$ \\
\hline BPNN & $1.145 \pm 0.525$ & $0.991 \pm 0.008$ & $21.435 \pm 2.930$ \\
\hline MNLR & $3.452 \pm 1.808$ & $0.938 \pm 0.035$ & $0.013 \pm 0.005$ \\
\hline
\end{tabular}

TABLE I. COMPARISON OF HANDGRIP FORCE PREDICTION (TRAINING DATASET)

\begin{tabular}{|c|c|c|c|}
\hline Method & $\operatorname{Mean}_{R M S} \pm$ S.D.RMSE $(k f g)$ & Mean $_{C C} \pm$ S.D..$_{C C}$ & $\operatorname{Mean}_{C T} \pm$ S.D..$_{C T}(s)$ \\
\hline BPNN & $1.207 \pm 0.487$ & $0.990 \pm 0.005$ & $0.008 \pm 0.001$ \\
\hline MNLR & $3.620 \pm 1.890$ & $0.931 \pm 0.041$ & $0.006 \pm 0.001$ \\
\hline
\end{tabular}

Table 2: Comparison of handgrip force prediction (Testing dataset)

As shown in Table 1, BPNN is about $66.831 \%$ better than MNLR at prediction accuracy. When two trained models are applied to testing datasets, the Mean $_{R M S E}$ value decreases by nearly $5.137 \%$ (BPNN) and $5.137 \%$ (MNLR), respectively. The prediction accuracy of BPNN is still about $66.657 \%$ higher than MNLR in Table 2. From Table 1 and Table 2, BPNN outperforms MNLR at Correlation Coefficient in both training dataset and testing dataset.

It is can be seen from Table 1 that BPNN takes long time to train the data. But BPNN consumes the time almost as much as MNLR in testing dataset. The Mean ${ }_{C T}$ is about $0.008 \mathrm{~s}(\mathrm{BPNN})$ and $0.006 \mathrm{~s}$ (MNLR), respectively.

The same results are demonstrated on every participant. The measured and predicted handgrip force from one participant is depicted in Fig .6 - Fig .9. The black solid line indicates the force measured from the dynamometer, and the red solid line indicates the force predicted by BPNN and MNLR.

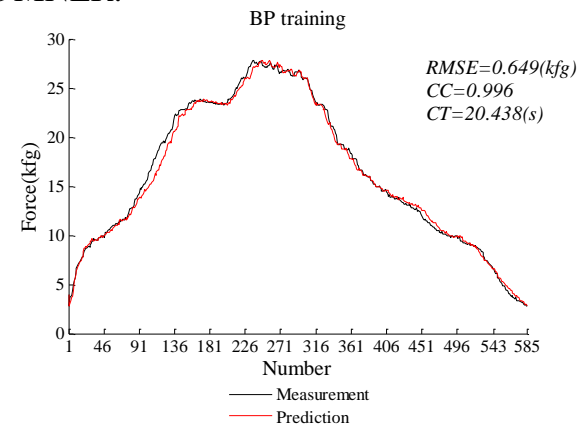

Figure 6. The results predicted by BPNN training. 


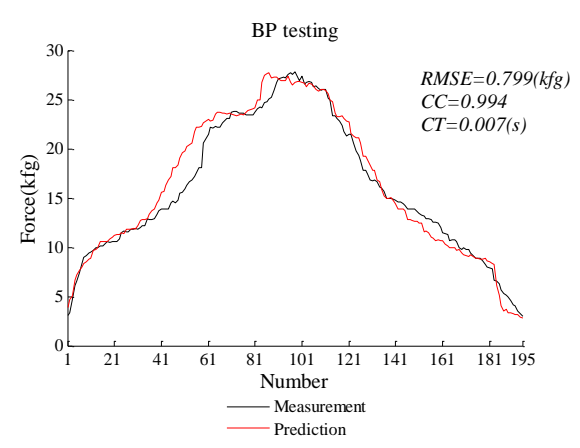

Figure 7. The results predicted by BPNN testing.

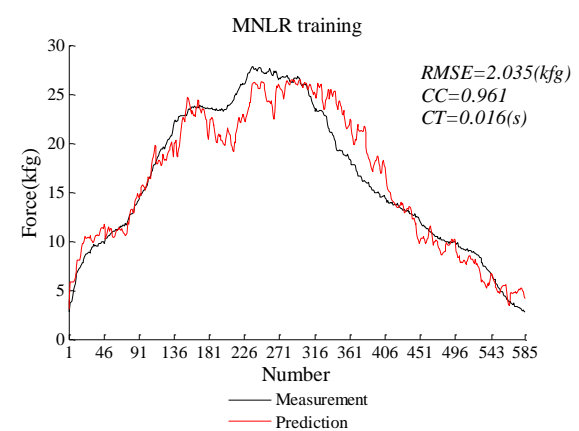

Figure 8. The results predicted by MNLR training.

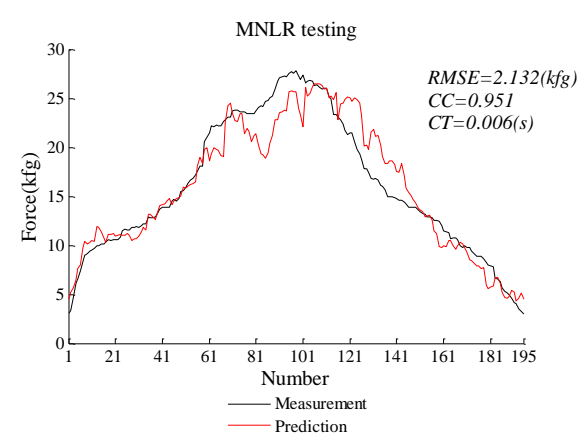

Figure 9. The results predicted by MNLR testing.

\section{CONCLUSIONS}

From the above experimental results, BPNN outperforms MNLR at prediction accuracy and Correlation Coefficient. If the control system of prostheses requires higher accuracy, BPNN is a suitable choice. As for consuming time, MNLR is better than BPNN When real time is required.

In future, how to shorten the consuming time of BPNN and improve the accuracy of MNLR should be studied. Then the control system with higher accuracy and realtime will be provided for prosthetic wrist.

\section{ACKNOWLEDGMENT}

This study is partly supported by the National Natura Science Foundation of China (61303137), the Fundamental Research Funds for the Central Universities (2014QNA5009) and the Specialized Research Fund for the Doctoral Program of Higher Education (20130101110148).

\section{REFERENCES}

[1] Charles G Burgar, Peter S Lum, Peggy Shor and H F Machievl Van der Loos, Development of robots for rehabilitation therapy: The
Palo Alto VA/Stanford experience, Journal of Rehabilitation Research and Development, vol. 37(6), pp. 663-673, 2000

[2] Masahiro Takaiwa and Toshiro Noritsugu, Development of wrist rehabilitation equipment using pneumatic parallel manipulator, IEEE International Conference on Robotics and Automation, Barcelona, pp. 2302-2307, 2005

[3] M.Akshay, W.T.Latt, H.G.Tan, U.X.Tan, C.Y.Shee and W.T.Ang Design and implementation of a mechatronic device for wrist and elbow rehabilitation, ICREAT, Singapore, 2007.

[4] Shahrul Naim Sidek, Ahmad Jazlan Haja Mohideen. Mapping of EMG signal to hand grip force at varying wrist angles. IEEE EMBS International Conference on Biomedical Engineering and Sciences, December, 2012.

[5] Sheikh Shanawaz Mostafa, Mohiuddin Ahmad, Md.Abdul Awal, Clench force estimation by surface electromyography for neural prosthesis hand, IEEE/OSA/IAPR Intenational conference on Informatics, Electronics \& Vision, pp. 505 - 510, 2012.

[6] Jakob R. Mathiesen, Mette F. Bøg, Ema Erkocevic, Marko J. Niemeier, Anne Smidstrup, Ernest N. Kamavuako, Prediction of grasping force based on features of surface and intramuscular EMG., 7th semester conference paper, pp. 1-9, 2010

[7] Marco J.M. Hoozemans, Jaap H. van Dieën, Prediction of handgrip forces using surface EMG of forearm muscles, Journal of Electromyography and Kinesiology, Vol. 15, pp. 358-366, 2005.

[8] Duque J, Masset D, Malchaire J, Evaluation of handgrip force from EMG measurements, Applied Ergonomics, Vol. 26, pp. 61-66, 1995

[9] Changmok Choi, Suncheol Kwon, Wonil Park, Hae-dong Lee, Jung Kim, Real-time pinch force estimation by surface electromyography using an artificial neural network, Medical Engineering \& Physics. Vol. 32, pp. 429-436, 2010.

[10] Marta C. Mora, Joaquín L. Sancho-Bru1, Antonio Pérez-González, Hand Posture Prediction using Neural Networks within a Biomechanical Model, International Journal of Advanced Robotic Systems, Vol. 9, pp. 1-11, 2012.

[11] T. W. Anderson, An introduction to Multivariate Statistical Analysis, 2nd Edition, John Wiley \& Sons, 1984

[12] Kleinbaum, David G., Kupper, Lawrence L., Muller, Keith E. Applied Regression Analysis and Multivariable Methods, 3rd Edition, Brooks/Cole, 1998.

[13] J.D.Horel, Complex principal component analysis: theory and examples, Journal of climate and applied meteorology, Vol. 23, pp. 1660-1673, 1984

[14] Neil H. Timm, Applied multivariate analysis, Springer, 2002.

[15] J. Edward Jackson, A User's Guide to Principal Components, Wiley, 1991.

[16] I. T. Jolliffe, Principal Component Analysis, Springer, 2002.

[17] Richard W. Bohannon, Anneli Peolsson, Nicola Massy-Westropp, Johanne Desrosiers, Jane Bear-Lehman, Reference values for adult grip strength measured with a Jamar dynamometer: a descriptive meta-analysis, Physiotherapy, Vol. 92, pp. 11-15, 2006.

[18] Virgil Mathiowetz, Karen Weber, Gloria Volland \& Nancy Kashman, Reliability and validity of grip and pinch strength evaluations, Journal of hand surgery, Vol. 9A, pp. 222-226, 1984.

[19] Haritha Srinivasan, Sauvik Gupta, Weihua Sheng, Heping Chen, Estimation of hand force from surface electromyography signals using artificial neural network, 10th world congress on intelligent control and automation. Beijing, pp. 584-589, 2012.

[20] Farid Mobasser; Keyvan Hashtrudi-Zaad, Rowing Stroke Force Estimation with EMG Signals using Artificial Neural Networks, 2005 IEEE Conference on Control Applications. Toronto, Canada, August, 2005.

[21] Nor Anija Jalaludin, Shahrul Na'im Sidek, Abu Ubaidah Shamsudin, Neuro-based thumb-tip force and joint angle modelling for development of prosthetic thumb control, International Journal of Advanced Robotic Systems, Vol. 10, pp. 1-8, 2013 